\title{
Robotic Worker in Construction Industry
}

\author{
Jingyi Mu and Shanshan Zhang* \\ Key Laboratory of Cold Region Urban and Rural Human Settlement Environment Science and Technology, Harbin Institute of Technology, \\ China
}

*Corresponding author: Shanshan Zhang, Key Laboratory of Cold Region Urban and Rural Human Settlement Environment Science and Technology, Ministry of Industry and Information Technology, School of Architecture, Harbin Institute of Technology, 66 West Dazhi Street, Nan Gang District, Harbin, 150001, China

\begin{abstract}
During the period when the COVID-19 occurred, China built two hospitals, "Huoshenshan" and "Leishenshan", in a short time, which created a miracle in the construction industry. For this miracle, construction robots are an indispensable part, which also has drawn attention from academic and technical circles to the construction industry. Construction robots have experienced more than one hundred years of development, and have experienced two generations regarding mechanical transmission and hydraulic pressure. The now robotized construction machinery is called the third generation, which is a milestone in the development of construction machinery. It can be controlled remotely, automatically and semi-automatically, and can perform a variety of operations, in which natural operation is the primary feature. With the development of robot technology, highly reliable and efficient construction robots have entered the market and have broad development and application prospects.
\end{abstract}

Keywords: Construction robots; Construction industry; Robotized construction machinery

\section{Introduction}

Construction robots have become a revolutionary construction technology. Robotized transformation of existing construction equipment is a shortcut to develop construction robot technology and quickly put it into applications, such as in excavators, bulldozers, road rollers, muck trucks [1-3]. Construction robots can be based on remote operation, autonomous navigation and obstacle avoidance, path planning and motion control, intelligent environment perception, unmanned driving and other technologies $[4,5]$. It is transformed to realize remote control, semi-autonomous, or even complete autonomy of related vehicle operations, reduce the workload of operators, optimize the working environment, improve operation safety and efficiency, and promote the standardization and refinement of construction operations [6-8]. With reference to this model, it is also possible to consider the remote operation transformation of lifting systems such as tower cranes and cranes, to prevent safety threats to construction personnel. Many robotic technologies commonly used are generic technologies [9], which have multiple applications in the construction industry. For example, mobile platforms such as unmanned aerial vehicles (UAV), wheeled/crawler robots, etc. can be used to carry Lidar and structured light cameras [10-12]. 3D vision and other environment perception devices, based on multi-source information fusion and simultaneous positioning with map creation (SLAM) and other environmental modeling technologies, can realize the independent mapping and 3D modeling of the internal and external structures of the building and the surrounding environment $[13,14]$; The use of unmanned aerial vehicle wells with SLAM technology to achieve earthwork excavation, waste removal and structural construction progress, and real-time monitoring of engineering quantities, provides a basis for the optimization and coordination of multiple equipment tasks in large-scale construction operations $[15,16]$. For another example, a universal mobile operating platform based on a manipulator and a mobile robot chassis is expected to replace manual operations such as masonry, plastering, leveling, polishing, weaving, paving, and drilling $[17,18]$. 


\section{Ceiling Drilling Robot}

The ceiling drilling robot overcomes the positioning problem through the combination of a camera and a laser, and the robot can achieve millimeter-level accuracy. The mobile construction robot of the ceiling drilling robot integrates all steps, and it only takes one step to drill 200,000 holes in the ceiling, with a high positioning accuracy. The biggest benefit of mobile construction robots is to increase productivity and efficiency. Another advantage is that the industrial vacuum cleaner on the robot platform can suck all the dust and debris and therefore protect the health of workers. In addition, it can also reduce the worker's risk of injury.
The professionally designed user interface eliminates the need for users to have a background in computer engineering, and can also customize the nLink app for Android tablets. In addition to drilling holes in the ceiling, the mobile construction robot can also perform other tasks, placing a plastic plug for screws, or a hook for ceilings or lights. Additionally, holes can be drilled in the wall or floor (Figure 1). In order to improve the accuracy and precision of distance measurement sensors based on laser triangulation, there are also some studies that have proposed a calibration model that correlates camera, lens, laser position and sensor position parameters with the robot base $[19,20]$.

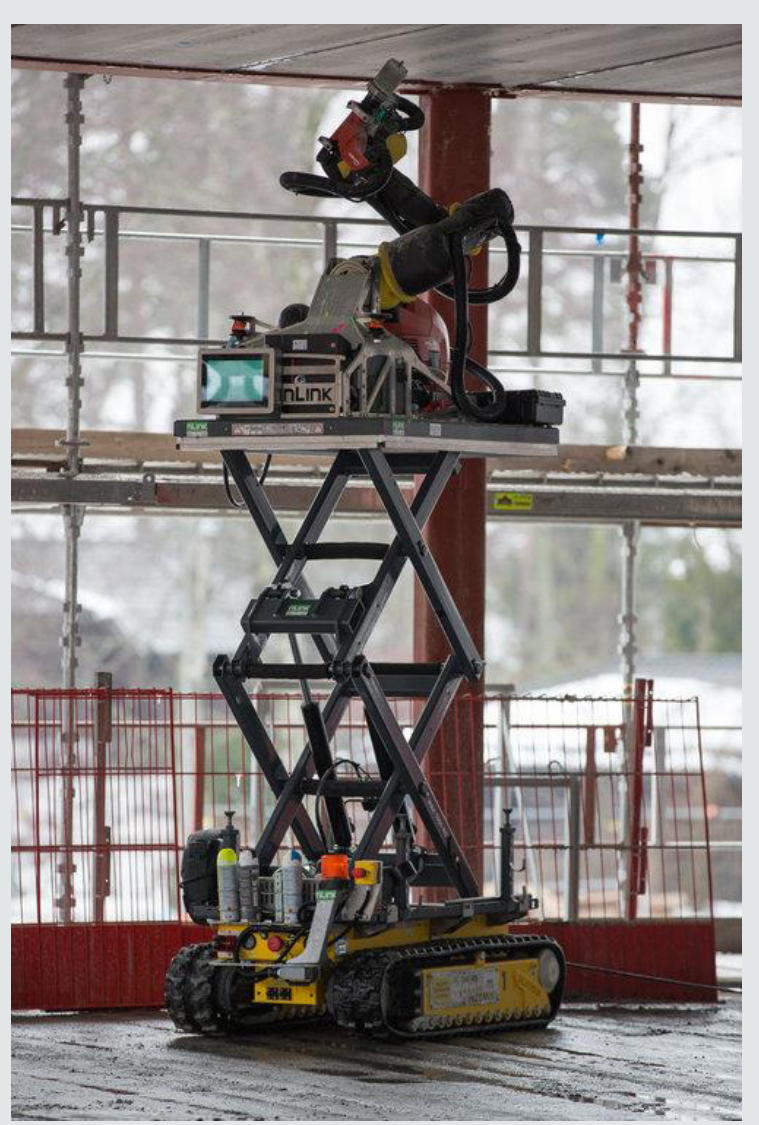

Figure 1: Examples of worldwide development on construction robots: ceiling drilling robot. Image Credit: image courtesy of nLink [20].

\section{Humanoid Robot Prototype HRP-5P}

HRP-5P can be used in large buildings such as the construction sites, shipyards and aircraft facilities. HRP-5P is a $182 \mathrm{~cm}, 101 \mathrm{~kg}$ humanoid robot with 37 degrees of freedom in the body: two on the neck, three on the waist, eight on the arms, six on the legs, and two on the hands. Except for hands, this represents the freest freedom of movement in the HRP series. HRP-5P, constructed with HRP series, adopts new hardware technology. In order to imitate the human body motion, in the absence of human freedom, the researchers ensured a wider range of joint movement in the hip and waist area (where multiple joints are concentrated). In this series, it has unparalleled physical capabilities. Its robot intelligence includes environmental measurement and object recognition, facilitated with whole-body motion planning and control, task description and execution management, and highly reliable systematic technology. The intelligence of the shell in this body has enabled autonomous plasterboard installation by robots, and HRP-5P can be used on construction sites. Using HRP-5P as a development platform, with the cooperation of industry and academia, it is expected to accelerate research and development, enabling the practical application of humanoid robots in construction sites and the assembly of large structures such as aircraft and ships (Figure 2) [21]. HRP-5P joint 
torque and speed doubled on average, using a high-output motor, adding cooling to the drive mechanism, and adopting a joint drive system with some joints with multiple motors. Therefore, the robot can perform tasks involving heavy loads, such as lifting gypsum boards from a stack. Each arm of HRP-5P extends horizontally and weighs $2.9 \mathrm{~kg}$. HRP-5P uses a head-mounted sensor, and the robot continuously obtains 3D measurements of the surrounding environment (frequency $0.3 \mathrm{~Hz}$ ). Even if the field of view is blocked by objects used at work, the stored and updated measurement results allow the execution of a walking plan while carrying a panel or correcting walking when the foot is slippery. HRP-5P can learn convolutional neural networks involving the use of a newly constructed object image database. The robot can detect 10 types of 2D object areas, even under low-contrast backgrounds or dim lights, with a high accuracy of $90 \%$ or more. By arranging a virtual test environment, setting up a virtual test environment for robot intelligence in the Choreonoid robot simulator, and monitoring the software regression for 24 hours, a highly reliable robot system can be constructed and the quality of large-scale software can be maintained.

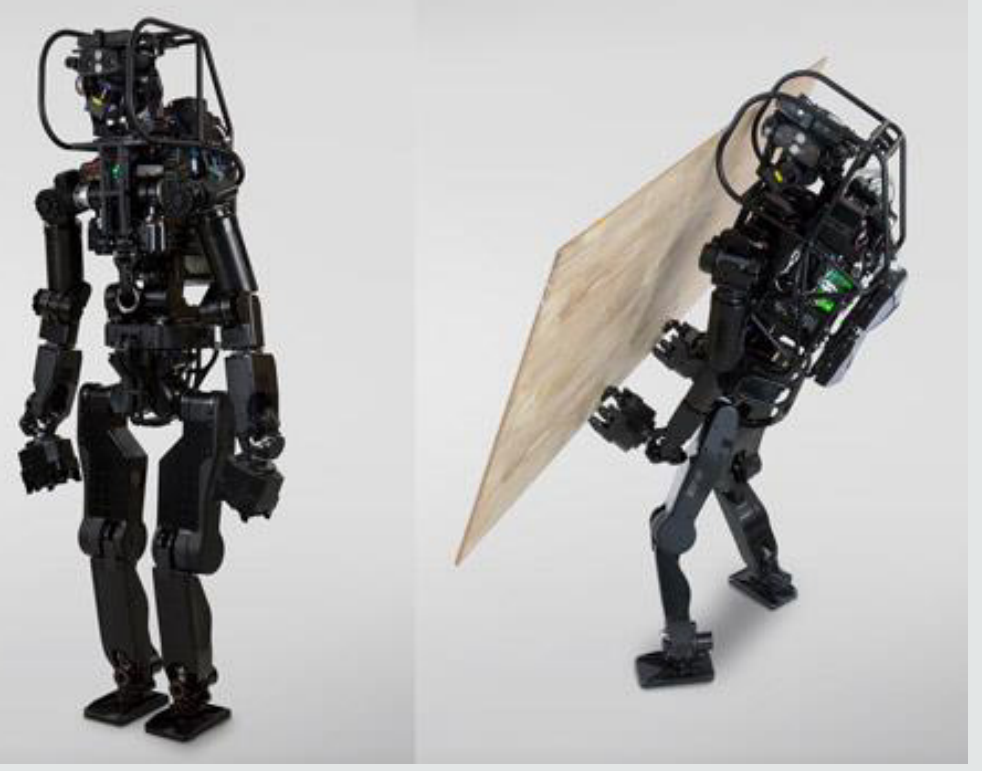

Figure 2: Examples of worldwide development on construction robots: humanoid robot prototype HRP-5P. Image Credit: image courtesy of Advanced Industrial Science and Technology (AIST) [21].

\section{Demolition Robot}

As people's requirements for the dynamic performance of crystal production in mechanical systems increase, it is necessary to accurately and quickly analyze and predict the dynamic characteristics of large and complex mechanical systems, and multi-body dynamics has also emerged. Brokk launched another new machine at Bauma in Munich. Prior to this, four new models were launched last year and the Brokk Smart Power ${ }^{\mathrm{TM}}$ series of machines were expanded. The new Brokk 70 is utilized to bring safe, mechanized and efficient demolition to the most enclosed spaces. Compared with its predecessor, Brokk 60, Brokk 70 is equipped with more than $100 \%$ additional demolition power, equipped with the latest innovative Brokk Smart PowerTM technology and a welladjusted hydraulic system to make it run more smoothly and faster. Nevertheless, it still maintains the same extremely compact size.
The weight does not exceed $560 \mathrm{~kg}$, which is suitable for passing through the narrowest door and can be transported in ordinary passenger elevators. The new Brokk BHB 105 circuit breaker weighs more than $100 \mathrm{~kg}$ and has advanced functions such as water spray to suppress dust and air-cooled thermal environments. For the first time, the new Brokk BDC 40 drum cutter brings true cutting capabilities to the smallest disassembly robot segment. The new Brokk G32 Demolition Fighter is a more effective soft demolition tool of choice. Very importantly, it still only runs on a 16-amp fuse. A disassembly robot that does not require manual disassembly. Analyze the rotation of a single arm, when the mechanical arm rotates at an angular velocity $\omega=100 \% / \mathrm{sec}$ at a constant speed, the hydraulic pressure on the mechanical arm is constant. As the radius of gyration increases, the center of mass velocity and acceleration gradually increase, which is consistent with the velocity and acceleration trend obtained by simulation (Figure 3) [22]. 


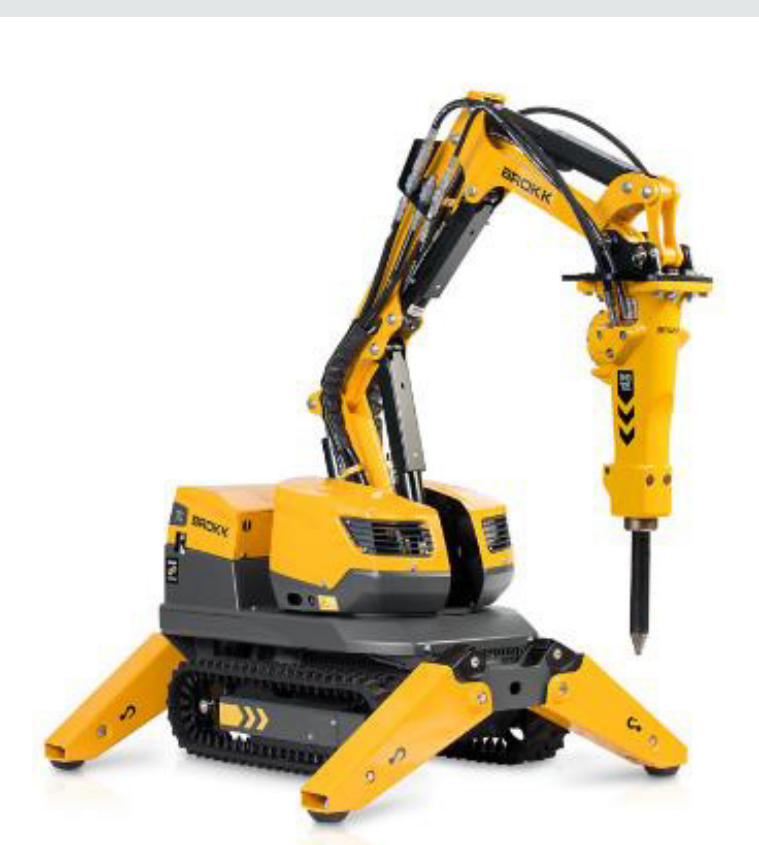

Figure 3: Examples of worldwide development on construction robots: demolition robot. Image Credit: image courtesy of BROKK [22].

\section{Conclusion}

Robots have advantages when performing building construction tasks. For example, a floor tile robot can accurately grasp and place floor tiles with a robotic arm to ensure the flatness of the floor tiles and the gap between the floor tiles, which consequently reduce the hollow rate of the floor tiles. The exterior wall spraying robot replaces the high-altitude operations in traditional buildings, greatly improving the safety, with 4 to 6 times of the traditional work efficiency. The floor cleaning robot can clean floor stones, debris and dust on the construction site in a dusty environment, and automatically empty the trash can. The cleaning rate is $90 \%$, and the construction efficiency is more than twice of manual operation. As an emerging technology with great development potential, construction robots are expected to realize "safer, more efficient, greener and smarter" informatization construction. The entire construction industry may take the opportunity to complete leapfrog development. Under the globally severe COVID-19 situation, building some much-needed buildings is an efficient and safe method. In the future, construction robots must have unlimited development potential, which can not only promote the promotion and application of construction robots in the construction industry, but also realize the rapid and safe development of the construction industry.

\section{References}

1. Liang CJ, Lundeen KM, McGee W (2019) A vision-based marker-less pose estimation system for articulated construction robots. Journal of Automation in Construction 104: 80-94.
2. Kim SH, Lee YS, Sun DI (2019) Development of bulldozer sensor system for estimating the position of blade cutting edge. Journal of Automation in Construction 106: 102890.

3. Singh AR, Patil Y, Delhi VSK (2019) Proceedings of the International Symposium on Automation and Robotics in Construction. Journal of IAARC Publications 36: 376-383.

4. Melenbrink N, Werfel J, Menges A (2020) On-site autonomous construction robots: Towards unsupervised building. Journal of Automation in Construction 119: 103312.

5. Pan M, Linner T, Pan W (2020) Influencing factors of the future utilisation of construction robots for buildings: A Hong Kong perspective. Journal of Building Engineering 30: 101220.

6. Oyekola P, Lambrache N, Mohamed A (2019) Design and Construction of an Unmanned Ground Vehicle.

7. Gheisari M, Esmaeili B (2019) Applications and requirements of unmanned aerial systems (UASs) for construction safety. Journal of Safety science 118: 230-240.

8. Makovetskaya E, Deniskina A, Krylov E (2019) Organizational optimization of construction processes by virtue of robotization. Journal of EDP Sciences 91: 02036.

9. Gharbia M, Chang-Richards AY, Zhong RY (2019) Robotic Technologies in Concrete Building Construction: A Systematic Review. Proceedings of the International Symposium on Automation and Robotics in Construction Journal of IAARC Publications 36: 10-19.

10. Howard J, Murashov V, Branche CM (2018) Unmanned aerial vehicles in construction and worker safety. Journal of American journal of industrial medicine 61(1): 3-10.

11. Gheisari M, Esmaeili B (2019) Applications and requirements of unmanned aerial systems (UASs) for construction safety. Journal of Safety science 118: 230-240.

12. Song G, Sun L, Yang X (2019) Design and Implementation of Selfbalancing and Navigation Robot Based on ROS System. Chinese Control and Decision Conference (CCDC) IEEE 5597-5602. 
13. Jiang G, Yin L, Jin S (2019) A simultaneous localization and mapping (SLAM) framework for 2.5 D map building based on low-cost LiDAR and vision fusion. Applied Sciences 9(10): 2105.

14. Tao C, Gao Z, Yan J (2020) Indoor 3D Semantic Robot VSLAM Based on Mask Regional Convolutional Neural Network. Journal of IEEE Access 8 : 52906-52916.

15. Kim P, Chen J, Kim J (2018) SLAM-driven intelligent autonomous mobile robot navigation for construction applications. Workshop of the European Group for Intelligent Computing in Engineering pp. 254-269.

16. Sasaki S, Hatakeyama S, Iwase M (2020) Construction of SLAM Algorithm for Window Cleaning Robot Moving Along Window Frame. Journal of Physics: Conference Series IOP Publishing 1487(1): 012023.

17. Gharbia M, Chang-Richards AY, Zhong RY (2019) Robotic Technologies in Concrete Building Construction: A Systematic Review. Proceedings of the
International Symposium on Automation and Robotics in Construction 36: $10-19$.

18. Ospishchev PI (2019) High-tech innovative robotized systems efficiency use in construction. IOP Conference Series: Materials Science and Engineering 698(6): 066009.

19. Idrobo Pizo GA, Motta JMST, Sampaio RC (2019) A calibration method for a laser triangulation scanner mounted on a robot arm for surface mapping. Journal of Sensors 19(8): 1783.

20. nLink (2018) Advanced mobile robotics

21. Advanced Industrial Science and Technology (2018) Development of a humanoid robot prototype, HRP-5P, capable of heavy labor.

22. Brokk (2019) Brokk-70.

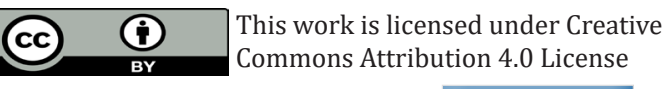

To Submit Your Article Click Here:

Submit Article

DOI: $10.32474 /$ ARME.2020.02.000149

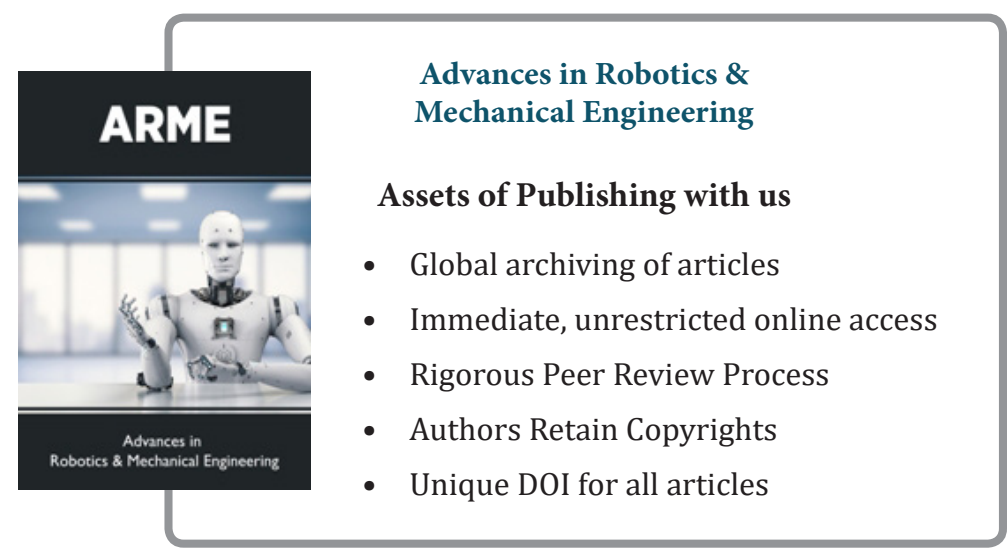

